\title{
Lymphangiography and thoracic duct embolization following unsuccessful thoracic duct ligation: Imaging findings and outcomes
}

\author{
Gregory J. Nadolski, MD, and Maxim Itkin, MD
}

\section{ABSTRACT}

Objective: To summarize the imaging findings and outcomes of thoracic duct (TD) embolization (TDE) performed in patients with chylous leaks persisting after TD ligation (TDL).

Materials and Methods: In this review of 50 patients (30 males and 20 females; median age, 54 years) referred for TDE following unsuccessful surgical TDL, records were reviewed for lymphangiographic findings, technical success of TDE, and outcome of TDE. Comparisons between groups were performed using the Fisher exact test.

Results: The causes of chylothorax were traumatic in 39 patients (78\%) and nontraumatic in $11(22 \%)$. Lymphangiography identified missed TDL in 30 patients $(60 \%)$ and complete TDL in 15 patients $(30 \%)$; however, in 12 of these 15 patients, collaterals around the ligation site supplying the leak could be identified. Incomplete ligation was observed in 4 patients $(8 \%)$. In 1 patient $(2 \%)$, a second TD was identified circumventing a complete ligation of the main TD. TDE was performed in 49 patients, and TD disruption was performed in 1 patient. Resolution of the chylous leak occurred in 45 patients $(90 \%)$. There were 3 minor complications that resulted in no clinical sequela.

Conclusions: TDE produced cessation of chylous leak in the majority of the patients with persistent chylothorax after surgical TDL. Missed ligation is the most common finding on lymphangiography in patients with failed TDL. These findings support the use of image-guided closure of TD leaks. (J Thorac Cardiovasc Surg 2018;156:838-43)

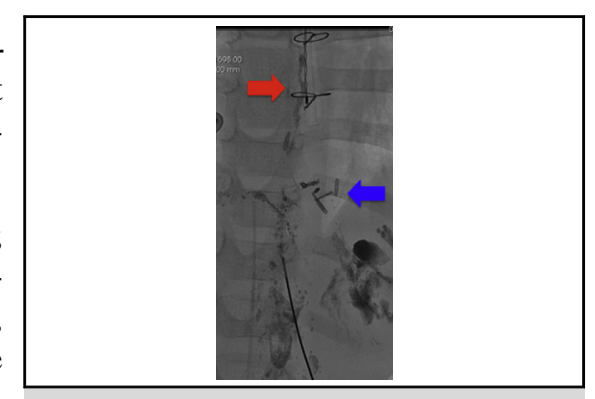

Missed TD with clips adjacent to the TD (blue arrow) and contrast in the central TD (red arrow).

\section{Central Message}

Persistent chylous leaks after failed thoracic duct ligation can be successfully treated with percutaneous thoracic duct embolization.

\section{Perspective}

Chylous leaks are an uncommon operative complication, and thoracic duct (TD) ligation (TDL) may be used to stop them. However, chylous leaks may persist after TDL. In patients referred for TD embolization (TDE) after unsuccessful TDL, the persistent leak was most often caused by missing the TD during ligation. TDE successfully resolved the chylous leak in $96 \%$ of these patients.

See Editorial Commentary page 844.

See Editorial page 836.
Chylous effusions, either spontaneous or traumatic, can result in severe protein malnutrition and electrolyte imbalances and may be ultimately fatal. Conservative management of chylous leaks consists of chest tube drainage, diet

From the Division of Interventional Radiology, Department of Radiology, University of Pennsylvania, Philadelphia, Pa.

Received for publication July 27, 2017; revisions received Feb 19, 2018; accepted for publication Feb 28, 2018; available ahead of print May 11, 2018.

Address for reprints: Gregory J. Nadolski, MD, Silverstein Hospital of the University of Pennsylvania, 3400 Spruce St, Philadelphia, PA 19104 (E-mail: gregory. nadolski@uphs.upenn.edu).

$0022-5223 / \$ 36.00$

Copyright (c) 2018 by The American Association for Thoracic Surgery

https://doi.org/10.1016/j.jtcvs.2018.02.109 modification (low-fat diet, nothing by mouth, and/or total parenteral nutrition) and intravenous infusion of octreotide. ${ }^{1}$ Unfortunately, conservative management can fail in up to $70 \%$ of cases. ${ }^{2}$

Traditionally, surgical management of chylous effusions with thoracic duct (TD) ligation (TDL) and pleurodesis is

- Scanning this QR code will take you to a supplemental video for the article.

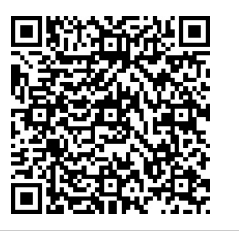




\section{Abbreviations and Acronyms \\ n-BCA $=N$-butyl cyanoacrylate \\ $\mathrm{TD}=$ thoracic duct \\ $\mathrm{TDE}=$ thoracic duct embolization \\ $\mathrm{TDL}=$ thoracic duct ligation}

performed in patients who fail conservative management. The reported success rates of TDL vary, depending on inclusion criteria and surgical technique, but can be as low as $67 \% .^{3} \mathrm{TD}$ embolization (TDE) is a minimally invasive alternative to TDL, with reported clinical success rates $>70 \%$ regardless of etiology. ${ }^{3,4}$ TDE comprises 2 parts, imaging with lymphangiography and intervention using percutaneous transabdominal lymphatic embolization. The ability to identify the anatomy of the lymphatic system and location of the leak potentially improves the outcomes of treatment. TDE attempted following unsuccessful TDL has been shown to be successful in small numbers of patients included in larger series reporting TDE outcomes. $^{4,5}$

The objectives of this study were to characterize the causes of persistent chylous leaks following TDL detected on lymphangiography, and to evaluate the efficacy of TDE following unsuccessful TDL.

\section{MATERIALS AND METHODS}

A Health Insurance Portability and Accountability Act (HIPAA) waiver and Institutional Review Board approval were granted before the start of this study. We performed a retrospective review of patients referred for TDE following unsuccessful surgical TDL, defined as continued symptomatic chylous leak(s) following attempted surgical ligation, between 2003 and 2016 using a prospectively recorded interventional radiology quality assurance database (HI-IQ; ConexSys, Lincoln, RI) to identify patients. Medical and procedural records were reviewed for mechanism of surgical failure, lymphangiographic findings, and technical and clinical success of TDE (Table 1).

\section{Pedal Lymphangiography}

Pedal lymphangiography was performed using a technique that has been described in detail previously. ${ }^{3,4,6}$ In brief, to opacify the pedal lymphatic vessels and facilitate lymphatic vessel dissection, isosulfan blue $1 \%$ (Lymphazurin; US Surgical, Norwalk, Conn) suspended in lidocaine 1\% was injected into the dorsal web spaces between the toes. Then, through a transverse incision on the dorsum of the foot, a lymphatic vessel was dissected free of soft tissue and cannulated using a 30-gauge needle (Cook, Bloomington, Ind). Ethiodized oil (Lipiodol; Guerbet, Bloomington, Ind) was then injected (median volume, $11 \mathrm{~mL}$; range, 6-20 mL) using a dedicated lymphangiogram pump (Cordis; Johnson and Johnson, Miami Lakes, Fla). At the completion of the injection, $20 \mathrm{~mL}$ of normal saline was injected into the lymphatic vessels to flush contrast out of the leg and facilitate opacification of the TD and cisterna chyli.

\section{Intranodal Lymphangiography}

The intranodal lymphangiography technique was performed as described previously. ${ }^{5,7}$ In brief, ultrasound of the inguinal and medial upper thigh was performed using a linear transducer (L12-5; Phillips, Andover, Mass) to identify a lymph node, which was directly accessed
TABLE 1. Demographic information

\begin{tabular}{lc}
\hline \multicolumn{1}{c}{ Characteristic } & Value \\
\hline Sex, $\mathrm{n}$ & \\
Male & 30 \\
Female & 20 \\
\hline Age, $\mathrm{y}$, median (range) & $54(13-82)$ \\
Etiology of chylothorax, $\mathrm{n}(\%)$ & $39(78)$ \\
Traumatic & 13 \\
Esophagectomy & 9 \\
Lung resection & 5 \\
Cardiac/thoracic aortic surgery & 3 \\
Neck dissection & 9 \\
Other thoracic surgery & $11(22)$ \\
Nontraumatic & 5 \\
Idiopathic chylothorax & 2 \\
Gorham disease & 1 \\
Generalized lymphatic anomaly & 2 \\
Idiopathic chylothorax/chyloptysis & 1 \\
Idiopathic chylothorax/chylopericardium & \\
Site of chylous leak, $\mathrm{n}$ & $45 / 26 / 14 / 4$ \\
Chylothorax (total/right/left/bilateral) & 2 \\
Unilateral neck & 1 \\
Pericardial & 2 \\
Chylopthysis & \\
\hline
\end{tabular}

under real-time ultrasound guidance with a 25-gauge spinal needle (BD, Franklin Lakes, NJ). The spinal needle was preassembled before nodal access with the stylet removed and the needle attached to a short segment of intravenous tubing and a 3-mL polycarbonate syringe (Merit Medical, South Jordan, Utah) containing an oil-based contrast agent (Lipiodol; Guerbet, Bloomington, Ind). Once the initial injection was observed under fluoroscopy to confirm proper positioning of the needle, contrast was injected by hand at a rate of approximately $1 \mathrm{~mL}$ per 5 minutes (median, $12 \mathrm{~mL}$; range, $8-40 \mathrm{~mL}$ ), and progression of the contrast was followed by intermittent fluoroscopy. The TD was then accessed and embolized as described below.

\section{TDE Procedures}

After identification of a target lymphatic (afferent or efferent lymphatic from the cisterna chyli), the target was accessed percutaneously using a 21or 22-gauge, 15- to 25-cm-long Chiba needle (Cook) via a transabdominal approach. A stiff 0.018-inch guidewire (V18 Control; Boston Scientific, Natick, Mass) was advanced through the needle into the lymphatic system. A 65-cm Rapid Transit microcatheter (Cordis) was then advanced into the lymphatics over the wire. Iodinated water-soluble contrast (Isovue 370; Bracco, Princeton, NJ) was injected through the microcatheter to identify the chylous leak. TDE was then performed.

Over the course of the study period, different embolization materials were used to occlude the TD (Table 2). Platinum embolization coils (Micronester; Cook) were used to pack the lumen of the TD and impede flow. In addition, liquid embolics, $N$-butylcyanoacrylate (n-BCA) glue (TrueFill; Cordis) and Onyx (Micro Therapeutics, Irvine, Calif), alone or in combination with intravascular coils, were used to occlude the TD. When used, True-Fill glue was diluted 1:1 with Ethiodol to delay polymerization; Onyx was used according to the manufacturer's instructions. In some cases where the injection of the glue was considered risky, embolization with autologous blood was performed. If the TD could not be catheterized, intentional fenestration/disruption of the upper abdominal lymphatics was performed at the discretion of the operating physician. If TDE was technically successful but failed clinically, repeat embolization was performed. 
TABLE 2. Procedure characteristics

\begin{tabular}{lc}
\hline \multicolumn{1}{c}{ Characteristic } & Value \\
\hline TDE procedures per patient (total $\mathrm{n}=57), \mathrm{n}$ & \\
1 & 39 \\
2 & 4 \\
3 & 2 \\
4 & 2 \\
Findings on initial lymphangiography, $\mathrm{n}(\%)$ & \\
Missed TD ligation & $30(60)$ \\
Complete TD ligation & $15(30)$ \\
Incomplete TD ligation & $4(8)$ \\
Double TD & $1(2)$ \\
Lymphangiogram success & $(100)$ \\
Pedal lymphangiogram & 28 \\
Intranodal lymphangiogram & 22 \\
Successful cannulation of TD & $50(100)$ \\
TDE & 49 \\
Coils and glue & 32 \\
Glue only & 13 \\
Onyx & 2 \\
Blood patch & 2 \\
TD disruptions & 1 \\
\hline
\end{tabular}

$T D E$, Thoracic duct embolization; $T D$, thoracic duct.

\section{Characterization of TDL}

The lymphangiographic appearance of the post-ligation TD was categorized as (1) missed, when the TD was patent without overlying surgical clips or narrowing from suture ligation; (2) complete, when ligation resulted in abrupt occlusion of the TD; or (3) incomplete, when the TD was focally narrowed but allowed contrast to pass through to the site of the leak. Some patients exhibited the presence/development of the lymphatic collaterals/parallel channels supplying the leak (Video 1).

\section{Clinical Follow-up}

After the procedure, the output from pleural/pericardial drainage tubes was monitored, and once decreased to $<200 \mathrm{~mL} /$ day, a food challenge test was administered. If after the food challenge test, drainage output did not

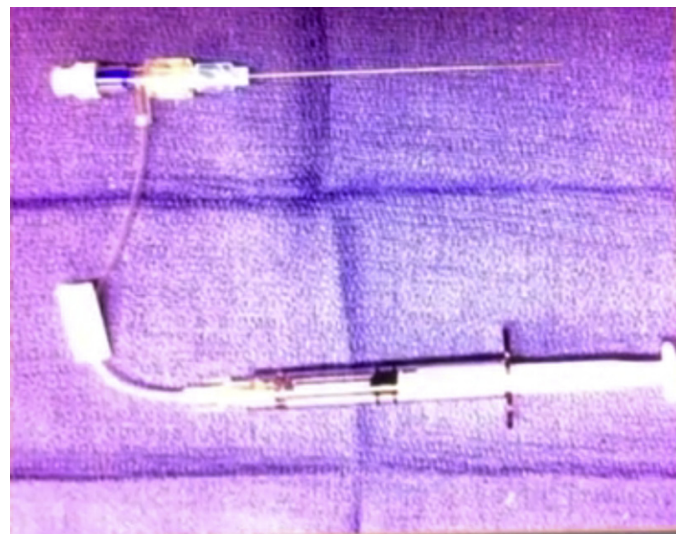

VIDEO 1. Discussion of the procedural details of thoracic duct embolization and variant anatomy that may be encountered in patients with unsuccessful thoracic duct ligation with persistent chylous leak. Video available at: https://www.jtcvs.org/article/S0022-5223(18)30936-X/fulltext. increase and/or turn chylous, the diet was advanced, and the drains were removed.

The technical success of TDE was defined as cannulation of the TD and delivery of embolic material if indicated to treat a chylous leak. Clinical success was defined as resolution of the chylous leak permitting removal of drains within 7 days.

\section{Statistical Methods and Analysis}

Patients were categorized by mechanism of chylous leak (traumatic vs nontraumatic) and lymphangiographic findings (missed/incomplete vs complete ligation of TD). Statistical analysis was performed using Stata version 13.1 (StataCorp; College Station, Tex). Categorical variables (eg, clinical success) were analyzed using the Fisher exact test. The significance level was set at $P<.05$.

\section{RESULTS}

Fifty patients (30 males and 20 females; median age, 54 years; range, 13-82 years) were included in this study (Figure 1). The causes of chylothorax were traumatic in 39 patients $(78 \%)$ and nontraumatic in $11(22 \%)$. Patient demographics and clinical presentations are summarized in Table 1. Procedural details of the TDEs are summarized in Table 2.

Lymphangiography was technically successful in all cases. TDE was performed via pedal lymphangiography

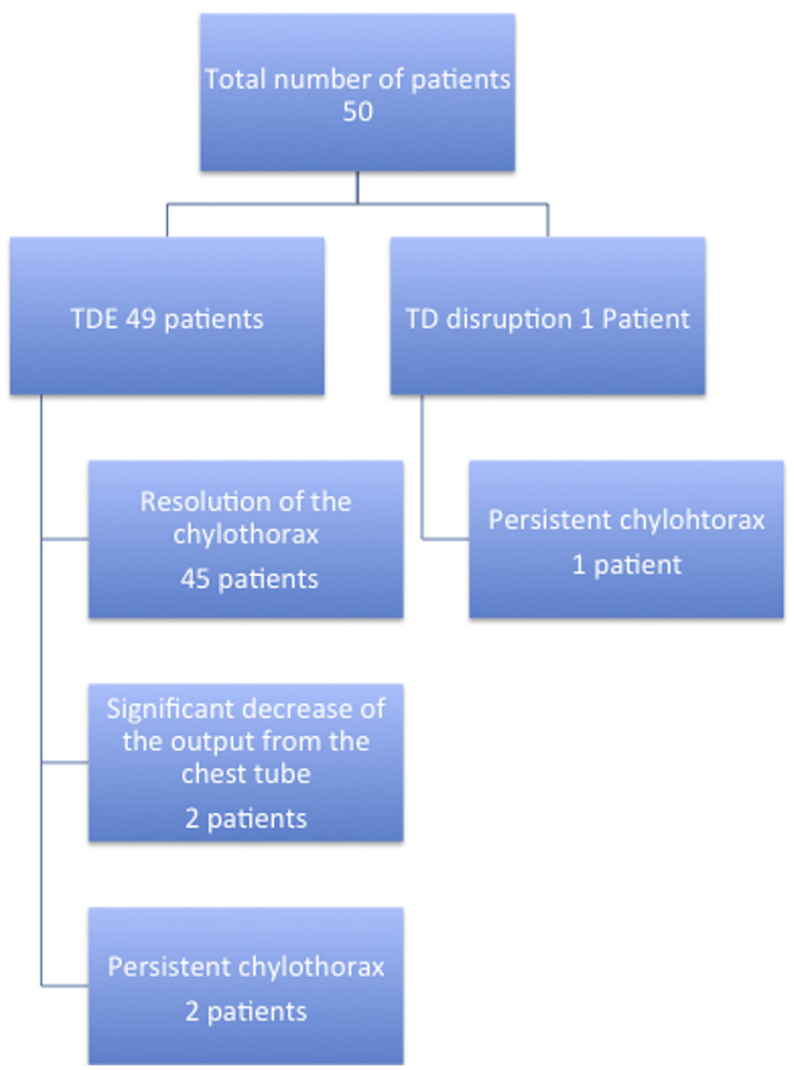

FIGURE 1. Flow diagram of included subjects regarding treatment type and outcome of chylous leak. TDE, Thoracic duct embolization; $T D$, thoracic duct. 
in 28 patients $(56 \%)$ and intranodal lymphangiography in 22 patients $(44 \%)$.

Catheterization of the TD was technically successful in all patients. TD injection through the microcatheter identified missed TDL in 30 patients $(60 \%)$ and complete TDL in 15 patients $(30 \%)$; however, in 12 of these 15 patients, collaterals around the ligation site supplying the leak could be identified (Figure 2). Incomplete ligation was observed in 4 patients $(8 \%)$ (Figure 3$)$. In 1 patient $(2 \%)$, a second TD was identified, circumventing complete ligation of the main TD.

TDE was performed in 49 patients, with coils in combination with n-BCA glue in 26 patients, n-BCA glue only in 12 patients, the Onyx embolization system in 2 patients, and autologous blood in 2 patients. In 1 patient, after successful TD cannulation, the access was lost and could not be regained. In this patient, TD disruption was performed as described by Cope et al. ${ }^{3}$ The overall technical success rate was $98 \%$.

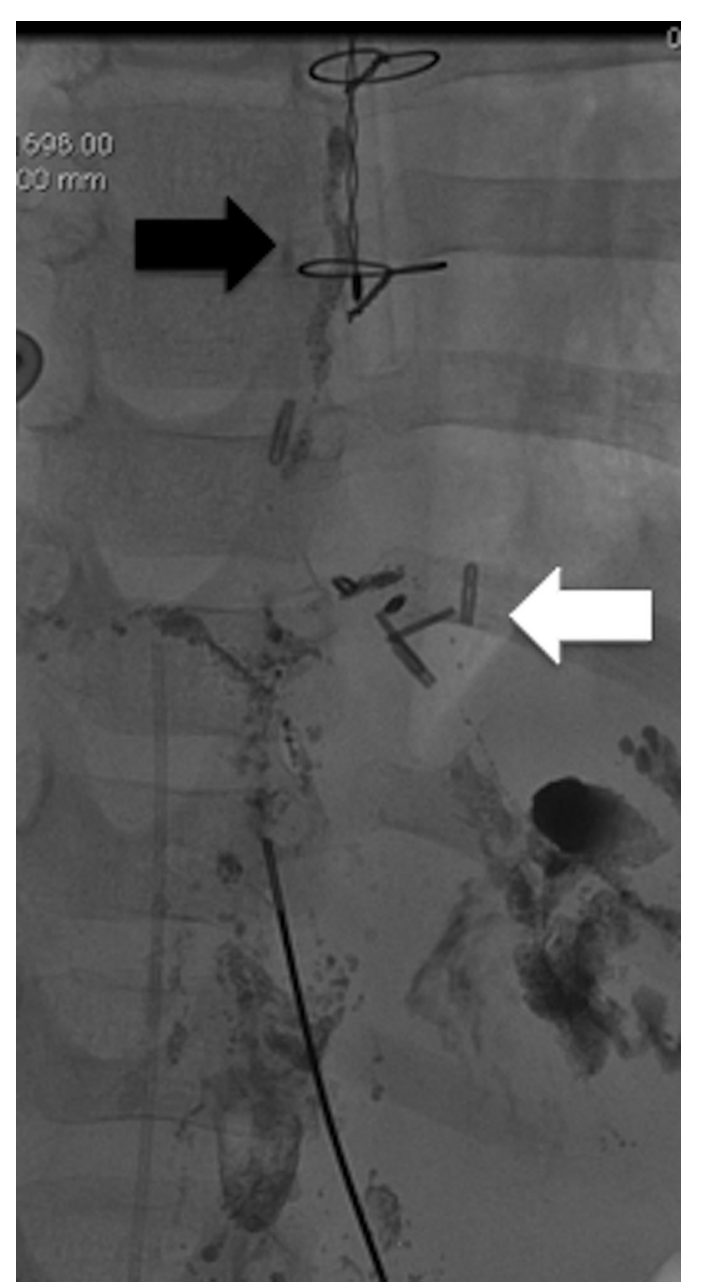

FIGURE 2. Fluoroscopic image demonstrating missed thoracic duct ligation. Multiple surgical clips are adjacent to the thoracic duct (white arrow). Contrast passes freely into the more central thoracic duct (black arrow).

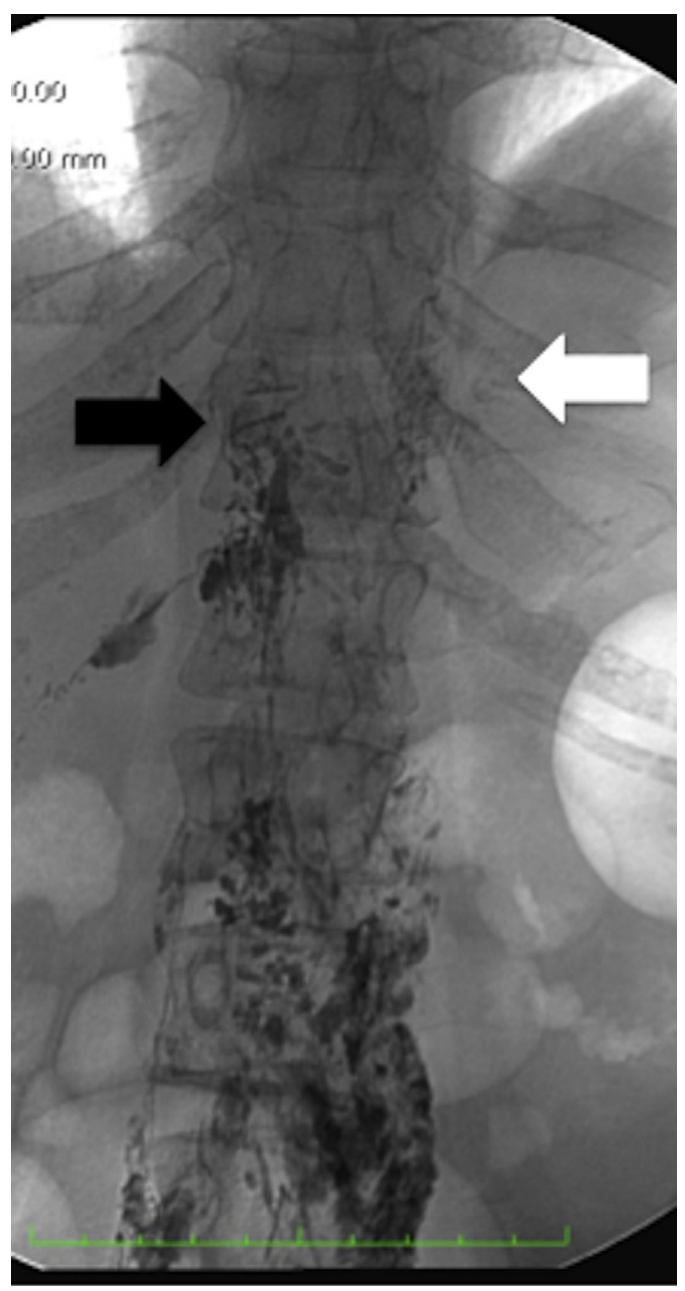

FIGURE 3. Fluoroscopic image demonstrating complete occlusion of the thoracic duct at the diaphragmatic hiatus from the surgical ligation (black arrow) with collaterals around the site of surgical ligation (white arrow), which allows lymph to enter the thoracic cavity.

Resolution of the symptoms occurred in 45 patients $(96 \%)$. In 3 patients, the chylothorax persisted despite technically successful TDE. In all of these 3 patients, TDE was performed at the beginning of the early stages of development of the embolization technique. Two patients $(4 \%)$ exhibited a significant reduction of chest tube output. One of these patients died at 2 weeks after the TDE, before chest tube removal, due to an unrelated cause, and the other was lost to follow-up.

Resolution of chylous leak did not differ significantly by etiology of chylothorax. Following TDE, 36 patients with postsurgical leaks $(92 \%)$ ) experienced resolution, compared with 9 patients with spontaneous leaks $(82 \% ; P=.30)$. Resolution of chylous leak did not differ based on the lymphangiographic findings or the embolic approach used. Clinical success was more often associated with embolization using the combination of coils and glue (30 of $32 ; 94 \%$ ) compared with other methods (15 of 18 ; 
$82 \%$ ), but this difference did not reach statistical significance $(P=.33)$. Likewise, clinical success in patients with missed or incomplete ligation (30 of $34 ; 88 \%$ ) was similar when compared to patients with complete ligation of the TD (14 of $15 ; 93 \% ; P=.99)$.

A total of 3 minor complications occurred in the 61 procedures $(5 \%)$. In 1 patient, access to the TD was lost due to respiratory motion, and access could not be regained. Intentional disruption of the lymphatics was performed in this patient, which ultimately did not resolve the chylothorax. In another patient, a coil was deployed in the retroperitoneal soft tissues with no clinical consequences. No attempt was made to retrieve the coil. The third patient became apneic after administration of moderate sedation. The patient responded to intravenous administration of $0.4 \mathrm{mg}$ naloxone without permanent adverse sequelae.

\section{DISCUSSION}

Here we have described our experience with percutaneous treatment of chylothorax and chyloptysis that persist following TDL. The treatment of the chylous leaks starts with a conservative approach that includes diet modification in an attempt to reduce the flow rate of the chyle from the intestinal lymphatics. ${ }^{8}$ In cases of high chest tube outflow and/or failed conservative treatment, interventional options are considered.

The safety of occlusion of the TD was more than a century ago. In 1907, DeForest ${ }^{9}$ opined that a wounded TD should be treated exactly as a bleeding blood vessel. Warschauer $^{10}$ even suggested that TD has to be ligated in all neck operation where it appears to be injured. Lee ligated TD in animals and was able to demonstrate two types of collateral circulation: to the right TD and to the azygos vein or its branches-left intercostal, and left lumbar veins. ${ }^{11}$ He concluded that non-functioning embryonic anastomoses exist between lymphatic and venous systems, and that these channels are open after ligation of the TD.

Peet $^{12}$ first described TDL to treat postsurgical chylothorax. The rationale for this approach was that interruption of the TD at its lower segment can stop the leak from the upper part of the duct. Since then, TDL has become the main approach to treating postsurgical chylothorax after failure of conservative treatment, with reported clinical resolution of the chylothorax in up to $91 \%$ of patients. ${ }^{2}$ However, the reported mortality after TDL is $10 \%$ and can reach $16 \%$ after surgical repair in high-risk patients. ${ }^{13-15}$

The anatomy of the central lymphatic system is often complex and extremely variable. ${ }^{16}$ Embryologically, the lymphatic system originates off veins as distinct lymphatic sacs demonstrated initially in the jugular-axillary area, with further development in the retroperitoneum, mediastinum, and pelvis. These primordial lymph sacs join together at different stages of development of the embryo and are separated from their venous origins with the exception of 2 to 3 connections between the jugular-axillary lymph sacs and venous angles. in 1915, Henry Davis published a central lymphatic system classification scheme that specified 9 subtypes. ${ }^{17}$ Later anatomic studies confirmed this variability and demonstrated multiple parallel ducts in $40 \%$ to $60 \%$ of cases. ${ }^{18-20}$

In this study, missed and incomplete TDL with the development of parallel ducts or collaterals were the most common causes of persistent chylothorax. TDL is guided primarily by anatomic landmarks owing to the difficulty of directly visualizing the TD during surgery. The reported rate of visualization of the site of leak and TD during TDL has been as low as $67 \% .{ }^{13}$ Furthermore, some surgical guidelines support TDL even in the absence of visualization of the leak or the TD. ${ }^{3}$ For these reasons, it is not surprising that the most common cause of the failed TDL was missing the TD. One advantage of TDE is its ability to visualize the anatomy of the lymphatic system and the source of the leak using lymphangiography, ${ }^{21,22}$ leading to improved outcomes. The use of a minimally invasive approach is also associated with lower postprocedure morbidity and mortality rates. ${ }^{4,6,23}$

One of the most surprising findings in this study is that chylothorax persisted in 15 cases of technically successful TDL. In the majority of these cases, collateral/parallel lymphatic vessels that either were present before ligation or developed after ligation were identified as the source of persistent leak on lymphangiography. As mentioned earlier, the significant variability of the central lymphatic anatomy that can be depicted as a network of multiple lymphatic vessels communicating with one another on different levels posses a challenge to occluding the TD without imaging guidance. The recent introduction of dynamic contrastenhanced magnetic resonance lymphangiography has allowed identification of multiple lymphatic pathways from the retroperitoneum toward the chest cavity. ${ }^{24}$ Interestingly, in 3 patients, we could not identify the presence of the collateral vessels while injecting the contrast into the cisterna chyli through an endovascular catheter; however, the chylothorax ceased immediately after injection of the n-BCA glue into the cisterna chyli in all 3 cases. It is possible that there were microscopic leaks or collaterals below the resolution of fluoroscopy.

The present study is not without limitations. First, the study is a retrospective review of patients referred to interventional radiology for embolization of chylous leaks after surgical ligation. The majority of the referrals came from outside our health system over a long period, and thus the exact technique for surgical ligation used in each patient is unknown, and techniques likely varied among the referring hospitals and over time. Furthermore, this pattern of referral limits the ability to identify a control population of patients who underwent TDL with resolution of chylothorax. In addition, the results of TDE are 
operator-dependent, and because all TDEs in this series were performed at a tertiary center with a large volume of lymphatic interventions, the results and outcomes might not be reproducible in all practice settings. Thus, future studies to directly compare TDE to surgical ligation of the TD are needed to directly compare these 2 interventions.

In conclusion, we have demonstrated that after failed TDL, missed or incomplete ligation is the most common finding on lymphangiography to explain the persistent chylous leak. Regardless of the etiology of chylothorax or cause of the persistent chylous leak, resolution can be achieved with TDE at equally successful rates. These observations support the use of TDE to treat symptomatic chylous leaks that persist after surgical ligation.

\section{Conflict of Interest Statement}

Authors have nothing to disclose with regard to commercial support.

\section{References}

1. Valentine VG, Raffin TA. The management of chylothorax. Chest. 1992;102: 586-91.

2. Cerfolio RJ, Allen MS, Deschamps C, Trastek VF, Pairolero PC. Postoperative chylothorax. J Thorac Cardiovasc Surg. 1996;112:1361-5; discussion 1365-6.

3. Cope C, Kaiser LR. Management of unremitting chylothorax by percutaneous embolization and blockage of retroperitoneal lymphatic vessels in 42 patients. J Vasc Interv Radiol. 2002;13:1139-48.

4. Itkin M, Kucharczuk JC, Kwak A, Trerotola SO, Kaiser LR. Nonoperative thoracic duct embolization for traumatic thoracic duct leak: experience in 109 patients. J Thorac Cardiovasc Surg. 2010;139:584-9; discussion 589-90.

5. Rajebi MR, Chaudry G, Padua HM, Dillon B, Yilmaz S, Arnold RW, et al. Intranodal lymphangiography: feasibility and preliminary experience in children. $J$ Vasc Interv Radiol. 2011;22:1300-5.

6. Cope C. Diagnosis and treatment of postoperative chyle leakage via percutaneous transabdominal catheterization of the cisterna chyli: a preliminary study. $J$ Vasc Interv Radiol. 1998:9:727-34.
7. Nadolski GJ, Itkin M. Feasibility of ultrasound-guided intranoda lymphangiogram for thoracic duct embolization. J Vasc Interv Radiol. 2012; 23:613-6.

8. Sriram K, Meguid RA, Meguid MM. Nutritional support in adults with chyle leaks. Nutrition. 2016;32:281-6.

9. De Forest HP VII. The surgery of the thoracic duct. Ann Surg. 1907:46:705-15.

10. Warschauer O. Ein beitrag zur chirurgie des ductus thoracicus [A contribution to surgery of the thoracic duct.] [in German]. Deutsche Zeitschrift für Chirurgie. 1912;119:422-7.

11. Lee FC. The establishment of collateral circulation following ligation of the thoracic duct. Bull Johns Hopkins Hosp. 1922;33:21.

12. Peet MM, Campbell KN. Massive chylothorax following splanchnicectomy: treatment with intravenous and instrasternal transfusions of chyle. Univ Hosp Bull Mich. 1943;9:2.

13. Bolger C, Walsh TN, Tanner WA, Keeling P, Hennessy TP. Chylothorax after oesophagectomy. Br J Surg. 1991;78:587-8.

14. Orringer MB, Bluett M, Deeb GM. Aggressive treatment of chylothorax compli cating transhiatal esophagectomy without thoracotomy. Surgery. 1988;104 720-6.

15. Sieczka EM, Harvey JC. Early thoracic duct ligation for postoperative chylothorax. J Surg Oncol. 1996;61:56-60.

16. Hsu MC, Itkin M. Lymphatic anatomy. Tech Vasc Interv Radiol. 2016;19: 247-54.

17. Davis HK. A statistical study of the thoracic duct in man. Am J Anat. 1915.

18. Akcali O, Kiray A, Ergur I, Tetik S, Alici E. Thoracic duct variations may complicate the anterior spine procedures. Eur Spine J. 2006;15:1347-51.

19. Phang K, Bowman M, Phillips A, Windsor J. Review of thoracic duct anatomical variations and clinical implications. Clin Anat. 2014;27:637-44.

20. Kausel HW, Reeve TS, Stein AA, Alley RD, Stranahan A. Anatomic and pathologic studies of the thoracic duct. J Thorac Surg. 1957:34:631-41.

21. Cope C. Percutaneous thoracic duct cannulation: feasibility study in swine. $J$ Vasc Interv Radiol. 1995;6:559-64.

22. Cope C. Percutaneous transabdominal embolization of thoracic duct lacerations in animals. J Vasc Interv Radiol. 1996;7:725-31.

23. Nadolski GJ, Itkin M. Thoracic duct embolization for non-traumatic chylous effusion: experience in 34 patients. Chest. 2013;143:158-63.

24. Dori Y, Zviman MM, Itkin M. Dynamic contrast-enhanced MR lymphangiography: feasibility study in swine. Radiology. 2014;273:410-6.

Key Words: chylothorax, thoracic duct ligation, thoracic duct embolization 